\title{
Mitigation of Linear and Nonlinear Impairments in High-Speed Optical Networks by using LDPC-Coded Turbo Equalization
}

\author{
Ivan B. Djordjevic, Member, IEEE, Lyubomir L. Minkov, and Hussam G. Batshon
}

\begin{abstract}
We study a turbo equalization scheme based on low-density parity-check (LDPC) coded turbo equalization (TE). This scheme is suitable for simultaneous: (i) suppression of intrachannel nonlinearities, (ii) chromatic dispersion compensation, and (iii) polarization-mode dispersion (PMD) compensation. LDPC coding is based on large girth $(g \geq 8)$ block-circulant codes, and maximum a posteriori probability (MAP) equalizer is based on Bahl-Cocke-Jelinek-Raviv (BJCR) algorithm. The ultimate channel capacity limits, assuming an independent identically distributed (i.i.d.) source are reported as well. In the presence of intrachannel nonlinearities the LDPC-coded TE provides almost $12 \mathrm{~dB}$ improvement over BCJR equalizer at BER of $10^{-8}$. For an NRZ system operating at $10 \mathrm{~Gb} / \mathrm{s}$ with residual dispersion of $11200 \mathrm{ps} / \mathrm{nm}$ and for differential group delay of $50 \mathrm{ps}$, the LDPC-coded TE is only $1 \mathrm{~dB}$ away from the i.i.d channel capacity. The efficiency of LDPC-coded TE in PMD compensation is demonstrated experimentally, with decoding performed off line.
\end{abstract}

Index Terms-Fiber-optics communications, intra-channel nonlinearities, chromatic dispersion, polarization-mode dispersion (PMD), turbo equalization, low-density parity-check (LDPC) codes.

\section{INTRODUCTION}

$\mathbf{O}$ PTICAL communication systems are evolving quickly to adapt to the ever-increasing demands of telecommunication needs, mostly noticeably witnessed by the growth in transmission capacity demands. To improve the overall transmission capacity, the number of channels has been increased through wavelength-division-multiplexing (WDM) or dense WDM (DWDM). Network operators consider $100 \mathrm{~Gb} / \mathrm{s}$ per DWDM channel transmission, yet the performance of fiberoptic communication systems operating at those data rates is degraded significantly due to several transmission impairments including intra- and inter-channel nonlinearities, polarizationmode dispersion (PMD), and chromatic dispersion [1]-[11]. These effects represent the current limiting factors in efforts to increase the capacity/speed, extend the transmission distance, and provide more flexible wavelength switching and routing capabilities in optical networks. To address the above challenges, novel advanced techniques and devices in modulation and detection, coding and signal processing should be developed.

\footnotetext{
Manuscript received February 6, 2008; revised June 10, 2008. This work is supported by the National Science Foundation under Grant IHCS-0725405.

The authors are with the University of Arizona, Department of Electrical and Computer Engineering, Tucson, AZ 85721, USA (e-mail: ivan@ece.arizona.edu).

Digital Object Identifier 10.1109/JSACOCN.2008.032908.
}

In this paper, we study the low-density parity-check (LDPC) coded turbo equalizer (TE) as a universal equalizer scheme for simultaneous suppression of fiber nonlinearities, for chromatic dispersion compensation and for PMD compensation. Notice that in our recent publications [8]-[10] we studied the efficiency of turbo equalization in dealing with intra-channel nonlinearities, chromatic dispersion and PMD, by observing scenarios in which each particular impairment dominate, although in practice those impairments act simultaneously. Moreover, we propose a new class of codes to be used in turbo equalization, based on large girth LDPC codes (the girth represents the shortest cycle in corresponding bipartite graph representation of a parity-check matrix). To facilitate the implementation at high-speed we prefer the use of structured LDPC codes [6] rather than random LPDC codes [12]. To reduce the performance loss of structured LDPC codes, compared to random ones, we use the girth [13] as the optimization parameter, and design the LDPC codes of girth8 and girth-10, as explained in Section III. The use of large girth LDPC codes increases the minimum distance, and decorrelates the extrinsic info in decoding process. The LDPCcoded turbo equalizer is composed of (i) Bahl-Cocke-JelinekRaviv (BCJR) algorithm [14] based equalizer (called here BCJR equalizer), and (ii) LDPC decoder is based on min-sumwith-correction-term algorithm [15]. The BCJR equalizer has the best immunity to the nonlinear intersymbol interference (ISI) due to intra-channel nonlinearities, residual chromatic dispersion and PMD, and provides soft bit-reliabilities (loglikelihood ratios, LLRs) required in LDPC decoding. To improve the overall bit-error ratio (BER) performance of turbo equalizer, we perform the iteration of extrinsic LLRs between LDPC decoder and BCJR equalizer, the procedure known as turbo equalization [16]. The extrinsic information transfer (EXIT) chart approach [17] is used to match the large-girth structured LDPC codes and BCJR equalizer. This scheme is able to operate in the presence of strong intra-channel nonlinearities, and provides large coding gains. For reasonable BCJR equalizer complexity it is able to compensate simultaneously for accumulated chromatic dispersion of $11200-\mathrm{ps} / \mathrm{nm}$, and differential group delay (DGD) of $50 \mathrm{ps}$ in an NRZ system operating at $10 \mathrm{~Gb} / \mathrm{s}$. The LDPC-coded turbo equalizer, based on girth-10 LDPC codes of rate 0.8 , provides almost $12 \mathrm{~dB}$ improvement over BCJR equalizer in an RZ system operating at $40 \mathrm{~Gb} / \mathrm{s}$, in the presence of intrachannel nonlinearities.

Given the fact that LDPC-coded turbo equalizer is an 


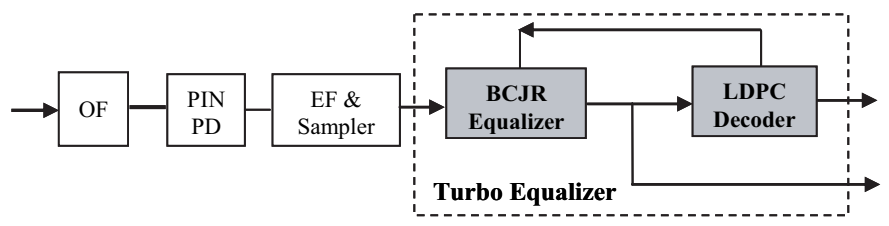

(a)

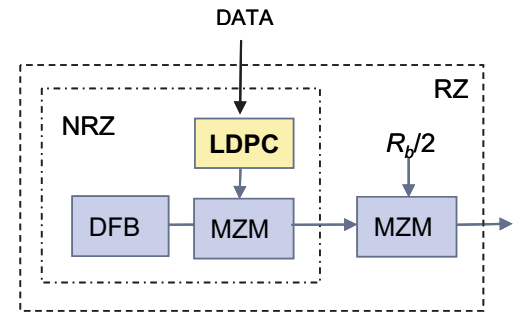

(b)

Fig. 1. (a) Receiver and LDPC-coded turbo equalizer architectures; and (b) transmitter configuration. MZM-Mach-Zhender modulator, DFB-distributed feedback laser, LDPC-LDPC encoder, OF-optical filter, PD-photodetector, EFelectrical filter.

excellent candidate to deal with nonlinear ISI, naturally arises the question about fundamental limits on channel capacity. In [26]-[29], we calculated the channel capacity of independent identically distributed (i.i.d.) information source (the achievable information rates) again observing different channel impairments separately. For completeness of presentation we also provide the channel capacity study when different degradation effects act simultaneously.

The paper is organized as follows. The LDPC-coded turbo equalization scheme is described in Section II. Design of large girth LDPC codes used in turbo equalizer is given in Section III. Sections IV-VI are devoted to the demonstration of efficiency of the LDPC-coded turbo equalizer in suppression of fiber nonlinearities (Section IV), for chromatic dispersion compensation (Section V), and for PMD compensation (Section VI). To verify the efficiency of LDPC-coded turbo equalizer in PMD compensation we perform the experiments. In Section VII we provide channel capacity study to see how closely channel capacity can be approached with reasonable complexity of turbo equalizer. In last Section, some important concluding remarks are provided.

\section{LDPC-Coded Turbo EQualization EQUALIZER DESCRIPTION}

As mentioned in Introduction, we will study LDPC-coded turbo equalization scheme, as a universal detection-decoding scheme that can be used simultaneously for: (i) suppression of fiber nonlinearities [8], (ii) PMD compensation [10], and (iii) chromatic dispersion compensation [9]. The LDPC-coded TE, shown in Fig. 1(a), is composed of two ingredients: (i) the BCJR algorithm based equalizer, and (ii) the LDPC decoder. Transmitter (shown in Fig. 1(b)) is based on standard RZ transmitter and an LDPC encoder. BCJR equalizer serves as nonlinear ISI canceller, reduces the BER down to the forward error correction (FEC) threshold, and provides accurate estimates of LLRs for LDPC decoder. To further improve BER performance we allow for iteration of extrinsic information between BCJR equalizer and LDPC decoder. The BCJR

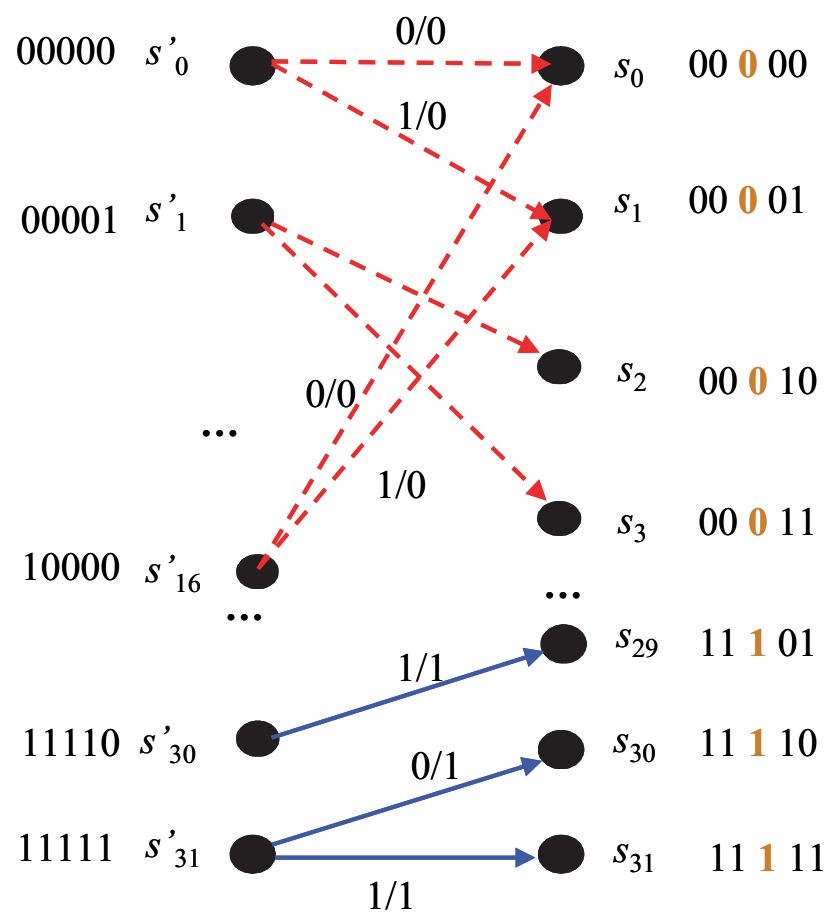

Fig. 2. Trellis description for $2 m+1=5$.

equalizer operates on a discrete dynamical trellis description of the optical channel, with memory equal to $2 m+1$, with $2 m$ being the number of bits that influence the observed bit from both sides. This dynamical trellis is uniquely defined by the set of previous state, the next state, in addition to the channel output. The state (the bit-pattern configuration) in the trellis is defined as $s_{j}=\left(x_{j-m}, x_{j-m+1}, \ldots, x_{j}, x_{j+1}, \ldots, x_{j+m}\right)=$ $x[j-m, j+m]$, where $x_{k} \in X=\{0,1\}$. An example trellis of memory $2 m+1=5$ is shown in Fig. 2. The trellis has $2^{5}=32$ states $\left(s_{0}, s_{1}, \ldots, s_{31}\right)$, each of which corresponds to a different 5-bit pattern. For the complete description of the trellis, the transition probability density functions (PDFs) $p\left(y_{j} \mid x_{j}\right)=p\left(y_{j} \mid s\right), s \in S$ can be determined from collected histograms $\left(y_{j}\right.$ represents the sample at the input of the BCJR equalizer that corresponds to the transmitted bit $u_{j}$, and $S$ is the set of states in the trellis). Because the collection of histograms is time consuming and sensitive to the precision, a better option would be to estimate conditional PDFs using instantons (or method of optimal fluctuations) to estimate far tails, and Edgeworth expansion to refine the middle part, as we proposed in [18].

The forward and backward recursion steps of BCJR equalizer are given in Figs. 3(a) and 3(b), respectively. Let $s^{\prime}=$ $x[j-m-1, j+m-1]$ represent the previous state, $s=$ $x[j-m, j+m]$ the present state, $x=\left(x_{1}, x_{2}, \ldots, x_{n}\right)$-the transmitted codeword, and $y=\left(y_{1}, y_{2}, \ldots, y_{n}\right)$-the received sequence of samples. The LLR, denoting the bit reliability, of $x_{j}(j=1,2, \ldots, n)$, can calculated by

$$
\begin{gathered}
L\left(x_{j}\right)=\max _{\left(s^{\prime}, s\right): x_{j}=0}^{*}\left[\alpha_{j-1}\left(s^{\prime}\right)+\gamma_{j}\left(s^{\prime}, s\right)+\beta_{j}(s)\right] \\
-\max _{\left(s^{\prime}, s\right): x_{j}=1}^{*}\left[\alpha_{j-1}\left(s^{\prime}\right)+\gamma_{j}\left(s^{\prime}, s\right)+\beta_{j}(s)\right]
\end{gathered}
$$

The dashed lines in Fig. 2 corresponds to transitions $\left(s^{\prime}, s\right)$ : $x_{j}=0$, and the solid lines to transitions $\left(s^{\prime}, s\right): x_{j}=1$. The 


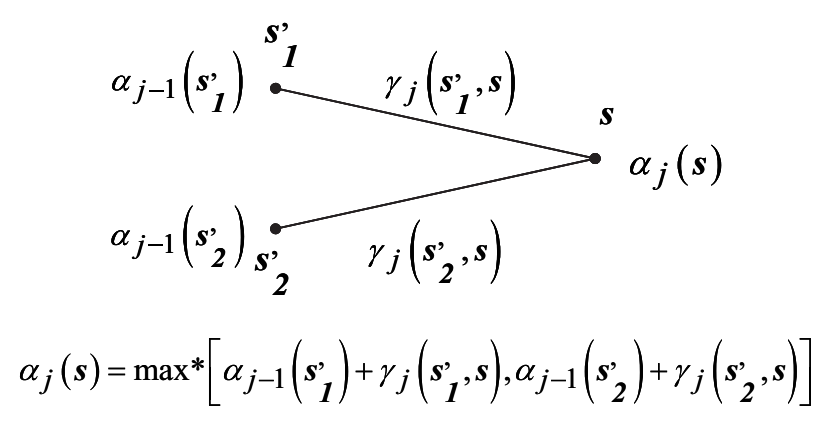

(a)

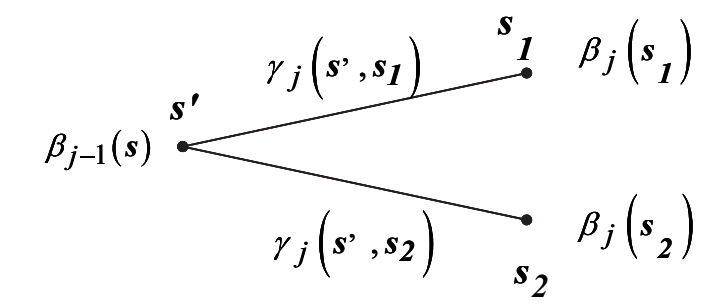

$\beta_{j-1}(s)=\max ^{*}\left[\beta_{j}\left(s_{1}\right)+\gamma_{j}\left(s^{\prime}, s_{1}\right), \beta_{j}\left(s_{2}\right)+\gamma_{j}\left(s^{\prime}, s_{2}\right)\right]$

(b)

Fig. 3. (a) The forward recursion step of BCJR equalizer, and (b) the backward recursion step of BCJR equalizer.

forward metric $\alpha_{j}(s)=\log p\left(s_{j}=s, y[1, j]\right)$, the backward metric $\beta_{j}(s)=\log p\left(y[j+1, n] \mid s_{j}=s\right)$, and the branch metric $\gamma_{j}\left(s^{\prime}, s\right)=\log \left[p\left(s_{j}=s, y_{j}, s_{j-1}=s^{\prime}\right)\right]$ in Eq. (1) can be determined respectively by

$$
\begin{gathered}
\alpha_{j}(s)=\max _{s^{\prime}}^{*}\left[\alpha_{j-1}\left(s^{\prime}\right)+\gamma_{j}\left(s^{\prime}, s\right)\right] \\
\beta_{j-1}\left(s^{\prime}\right)=\max _{s}^{*}\left[\beta_{j}(s)+\gamma_{j}\left(s^{\prime}, s\right)\right] \\
\gamma_{j}\left(s^{\prime}, s\right)=\log \left[p\left(y_{j} \mid x[j-m, j+m]\right) P\left(u_{j}\right)\right] .
\end{gathered}
$$

The $\max ^{*}$-operator is defined by $\max ^{*}(x, y)=$ $\log \left(e^{x}+e^{y}\right)$, and it is efficiently calculated by $\max (x, y)=\max (x, y)+c(x, y)$, where $c(x, y)$ is the correction factor, $c(x, y)=\log [1+\exp (-|x-y|)]$, commonly approximated or implemented using a look-up table. $p\left(y_{j} \mid x[j-m, j+m]\right)$ is obtained, as already explained above, by either collecting the histograms or by instanton-Edgeworth expansion method, and $P\left(x_{j}\right)$ represents a priori probability of transmitted bit $x_{j}$, which is either $1 / 2$ (because binary equally probable transmission is observed) for an existing transition from trellis given in Fig. 2, or zero for non-existing one. The initial forward and backward metrics values are set to

$$
\begin{array}{ll}
\alpha_{0}(s)=0, s=s_{0} & \alpha_{0}(s)=-\infty, s \neq s_{0} \\
\beta_{n}(s)=0, s=s_{0} & \beta_{n}(s)=-\infty, s \neq s_{0}
\end{array}
$$

where $s_{0}$ is an initial state. The key difference between the regular BCJR algorithm [14] (see also [19]) and the BCJR equalizer described here is therefore just in calculation of LLRs. The conventional BCJR algorithm, calculates LLRs of the input bits corresponding to the edges, while the BCJR equalizer calculates the LLRs of the output bits corresponding to the central bit of terminal states (see Fig. 2). Another important difference with respect to turbo equalization employed in magnetic recording systems [20] is that the BCJR equalizer operates on a trellis that includes both pre- and post-cursor ISI. The forward recursion step (Fig. 3(a)), and backward recursion step (Fig. 3(b)) are similar to that in the original log-domain BCJR algorithm [19].

The BCJR equalizer LLRs are forwarded to the softdecoding LDPC decoder implemented based on sum-productwith-correction-term algorithm [15]. To improve the overall performance of LDPC-coded turbo equalizer we allow the iteration of extrinsic LLRs between LDPC decoder and BCJR equalizer. For example, extrinsic LLRs for BCJR equalizer in $k$ th iteration are determined by

$$
L_{L D P C, e}\left(x_{j}^{(k)}\right)=L_{L D P C}\left(x_{j}^{(k)}\right)-L_{L D P C}\left(x_{j}^{(k-1)}\right),
$$

where $L_{L D P C}\left(x_{j}\right)$ is corresponding LLR of bit $x_{j}$, while indices $k$ and $k-1$ are used to denote the current and previous iterations. The extrinsic LLRs from (4) are used as a priori LLRs in the third line of (2) by

$L_{B C J R, a}\left(x_{j}\right)=\log \left[P\left(x_{j}\right)\right]=L_{L D P C, e}\left(x_{j}\right)$.

The LDPC codes are selected using the concept of EXIT charts [17], in a fashion similar to that we reported in [21]. To facilitate the implementation at high speed we prefer the use of structured quasi-cyclic LDPC codes of large girth. Such codes lead to encoders that can be implemented based on shiftregister and modulo-2 adders, and the complexity of decoder is low. One such class of LDPC codes is proposed in next Section.

\section{LARGE GIRTH LDPC CODES}

Based on Tanner's bound for the minimum distance of an LDPC code [22]

$d \geq\left\{\begin{array}{c}1+\frac{r}{r-2}\left((r-1)^{\lfloor(g-2) / 4\rfloor}-1\right), g / 2=2 m+1 \\ 1+\frac{r}{r-2}\left((r-1)^{\lfloor(g-2) / 4\rfloor}-1\right)+(r-1)^{\lfloor(g-2) / 4\rfloor}, g / 2=2 m\end{array}\right.$

where $g$ and $r$ denote the girth of the code graph and the column weight, respectively, and where $\mathrm{d}$ stands for the minimum distance of the code, it follows that large girth leads to an exponential increase in the minimum distance, provided that the column weight is at least 3. $(\lfloor\rfloor$ denotes the largest integer less than or equal to the enclosed quantity.) For example, the minimum distance of girth-10 codes with column weight $r=3$ is at least 10 . The structured LDPC codes introduced in this Section belong to the class of blockcirculant (BC) codes [23], also known as array codes [24]. Their parity-check matrix can be represented by

$$
H=\left[\begin{array}{ccccc}
I & P^{c_{0}} & P^{2 c_{0}} & \ldots & P^{(q-1) c_{0}} \\
I & P^{c_{1}} & P^{2 c_{1}} & \ldots & P^{(q-1) c_{1}} \\
\ldots & \ldots & \ldots & \ldots & \ldots \\
I & P^{c_{r-1}} & P^{2 c_{r-1}} & \ldots & P^{(q-1) c_{r-1}}
\end{array}\right]
$$

where $c_{i} \in\{0,1, \ldots, q-1\}(i=0,1, \ldots, r-1), I$ is the identity matrix of dimension $q$, and $P$ denotes the permutation matrix

$$
P=\left[\begin{array}{cccccc}
0 & 1 & 0 & 0 & \ldots & 0 \\
0 & 0 & 1 & 0 & \ldots & 0 \\
\ldots & \ldots & \ldots & \ldots & \ldots & \\
0 & 0 & 0 & 0 & \ldots & 1 \\
1 & 0 & 0 & 0 & \ldots & 0
\end{array}\right]
$$


The integers $c_{i}$ are to be carefully chosen according to Theorem $2.1 \mathrm{in} \mathrm{[13]} \mathrm{in} \mathrm{order} \mathrm{to} \mathrm{avoid} \mathrm{the} \mathrm{cycles} \mathrm{of} \mathrm{length} 2 k(k=$ 3 or 4). According to this theorem (see also [23]) the cycle of length $2 k$ exists if we can find the closed path in (6), denoted by $\left(i_{1}, j_{1}\right),\left(i_{1}, j_{2}\right),\left(i_{2}, j_{2}\right),\left(i_{2}, j_{3}\right), \ldots,\left(i_{k}, j_{k}\right),\left(i_{k}, j_{1}\right)$, such that

$$
c_{i_{1}} j_{1}+c_{i_{2}} j_{2}+\ldots+c_{i_{k}} j_{k}=c_{i_{1}} j_{2}+c_{i_{2}} j_{3}+\ldots+c_{i_{k}} j_{1} \bmod q,
$$

where $q$ is the dimension of the permutation matrix $P$, and should be a prime number. The pair of indices above denote row-column indices of permutation-blocks in (6) such that $l_{m} \neq l_{m+1}, l_{k} \neq l_{1}(m=1,2, \ldots, k ; l \in\{i, j\})$. In order to avoid the cycles of length $2 k, k=3$ or 4 , we have to find the sequence of integers $c_{i} \in\{0,1, \ldots, q-1\}(i=$ $0,1, \ldots, r-1 ; r<q)$ not satisfying the equation (7), which can be done either by computer search or in a combinatorial fashion. For example, to design the BC LDPC codes in [23] we introduced the concept of the cyclic-invariant difference set (CIDS). The CIDS-based codes come naturally as girth-6 codes, and to increase the girth we had to selectively remove certain elements from a CIDS. The design of LDPC codes of rate above 0.8 , column weight 3 and girth-10 using the CIDS approach is a very challenging, still an open problem. Instead, in this paper we solve this problem by developing an efficient computer search algorithm, which begins with an initial set. We add an additional integer at the time from the set $Q=\{0,1, \ldots, q-1\}$ (not used before) to the initial set $S$ and check if the equation (7) is satisfied. If the equation (7) is satisfied we remove that integer from the set $S$, and continue our search with another integer from set $Q$, until we exploit all the elements from $Q$. The code rate $R$ is lower-bounded by

$$
R \geq(n q-r q) / n q=1-r / n,
$$

and the code length is $n q$, where $n$ denotes the number of elements from $S$ being used. The parameter $n$ is determined by desired code rate $R_{0}$ by $n=r /\left(1-R_{0}\right)$. If desired code rate is set to $R_{0}=0.8$, and column weight to $r=3$, the parameter $n=5 r$.

Example: By setting $q=1129$, the set of integers to be used in (6) is obtained as $S=\{0,1,4,11,27,39,48,84,134,163,223$, $284,333,397,927\}$. The corresponding LDPC code has rate $R_{0}=1-3 / 15=0.8$, column weight 3 , girth- 10 and length $n q=15 \cdot 1129=16935$, which is about twice shorter than turbo-product code (TPC) proposed in [25]. In the example above, the initial set of integers was $S=\{0,1,4\}$. The use of a different initial set will result in a different set from that obtained above. In addition to this code we designed the LDPC $(8540,6835)$ code of rate 0.8 , girth- 8 and column weight 3 , and LDPC $(9276,6959)$ code of code rate 0.75 , girth-10, and column weight 3 . Notice that turbo-product codes of similar rates were considered in [25].

The results of simulations for an additive white Gaussian noise (AWGN) channel model are given in Fig. 4, where we compare the proposed LDPC codes against RS codes, concatenated RS codes, TPCs, and previously proposed LDPC codes. The girth-10 $\operatorname{LDPC}(24015,19212)$ code of rate 0.8 outperforms the concatenation $\mathrm{RS}(255,239)+\mathrm{RS}(255,223)$ (of rate 0.82$)$ by $3.35 \mathrm{~dB}$, and $\mathrm{RS}(255,239)$ by $4.75 \mathrm{~dB}$, both at

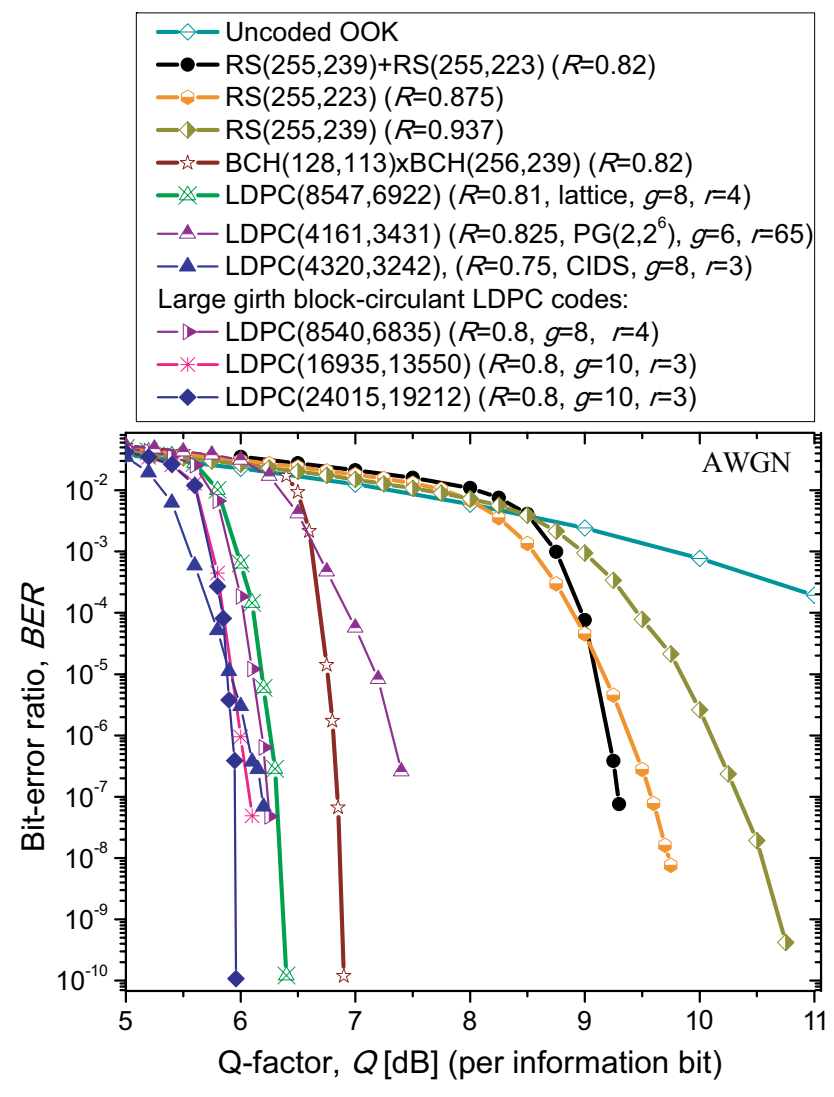

Fig. 4. Large girth block-circulant LDPC codes against RS codes, concatenated RS codes, TPCs, and previously proposed LDPC codes on an AWGN channel model.

BER of $10^{-7}$. The same LDPC code outperforms projective geometry (PG) $\left(2,2^{6}\right)$ based $\operatorname{LDPC}(4161,3431)$ (of rate 0.825 ) of girth- 6 by $1.49 \mathrm{~dB}$ at BER of $10^{-7}$, and outperforms CIDS based LDPC $(4320,3242)$ of rate 0.75 and girth-8 LDPC codes by $0.25 \mathrm{~dB}$. At BER of $10^{-10}$ it outperforms lattice based $\operatorname{LDPC}(8547,6922)$ of rate 0.81 and girth- 8 by $0.44 \mathrm{~dB}$, and $\mathrm{BCH}(128,113) \times \mathrm{BCH}(256,239) \mathrm{TPC}$ of rate 0.82 by $0.95 \mathrm{~dB}$. The net effective coding gain at $B E R$ of $10^{-12}$ is $10.95 \mathrm{~dB}$.

Given the description of LDPC-coded TE, and design of LDPC codes to be used in turbo equalization, in the following Sections we describe three applications of interest: (i) suppression of intra-channel nonlinearities (described in next Section), (ii) chromatic dispersion compensation (described in Section V), and PMD compensation (described in Section VI).

\section{SUPPRESSION OF INTRACHANNEL NONLINEARITIES VIA LDPC-CODED TURBO EQUALIZATION}

We are turning our attention to the description how to deal with intra-channel nonlinearities via LDPC-coded turbo equalization. For this purpose we developed a realistic fiberoptic communication system model based on the nonlinear Schroedinger equation that was solved using the split-step Fourier method. This model takes into account Kerr nonlinearities, stimulated Raman scattering, dispersion effects, amplified-spontaneous emission (ASE) noise, filtering effects, intersymbol interference, and linear crosstalk effects (for more details an interested reader is referred to [6]). It is well known that at $40-\mathrm{Gb} / \mathrm{s}$ and above the intra-channel nonlinearities, such 


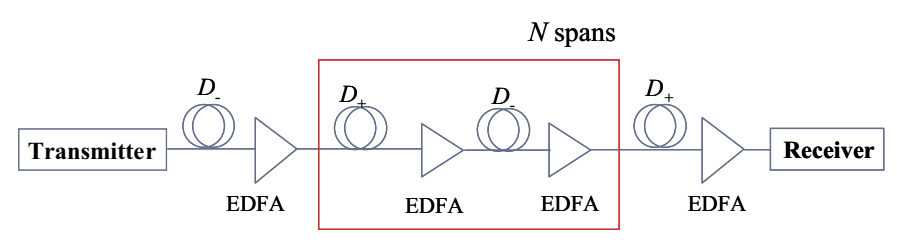

Fig. 5. Dispersion map under study.

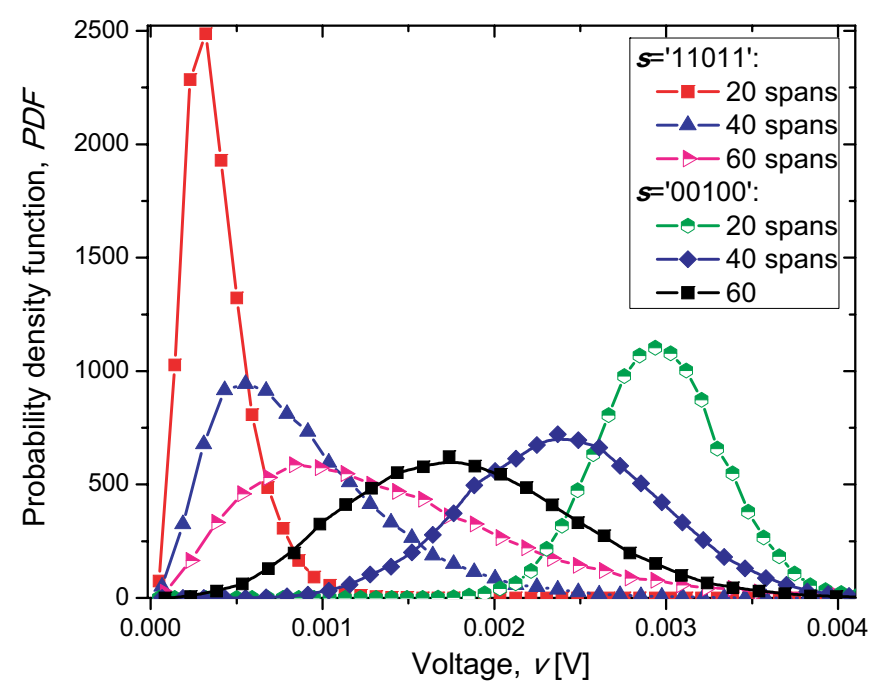

Fig. 6. Conditional PDFs $p\left(y_{j} \mid x[j-m, j+m]\right)$ against different number of spans estimated from simulator for RZ OOK.

as intra-channel four-wave mixing (IFWM) and intra-channel cross-phase modulation (IXPM), dominate over inter-channel nonlinearities. IXPM effects can be controlled by a proper dispersion map design, while IFWM cannot be eliminated using this approach. The dispersion map shown in Fig. 5 is selected in such a way that IFWM is the pre-dominant intrachannel nonlinear effect. The span length is set to $L=120 \mathrm{~km}$, and each span consists of $2 L / 3 \mathrm{~km}$ of D+ fiber followed by $L / 3 \mathrm{~km}$ of $\mathrm{D}$ - fiber. Pre-compensation of $-1600 \mathrm{ps} / \mathrm{nm}$ and corresponding post-compensation are also applied. The parameters of D+ and D- fibers, used in simulations, are given in Table 1. RZ modulation format of a duty cycle of 0.33 is observed, the extinction ratio is $14 \mathrm{~dB}$, and the launched power is set to $0 \mathrm{dBm}$. EDFAs with noise figure of $6 \mathrm{~dB}$ are deployed after every fiber section, the bandwidth of optical filter (modeled as super-Gaussian filter of eight order) is set to $3 R_{l}$ and the bandwidth of electrical filter (modeled as Gaussian filter) to $0.7 R_{l}$, with $R_{l}$ being the line rate (defined as the bit rate $(40 \mathrm{~Gb} / \mathrm{s})$ divided by a code rate). The line rate (defined above) is appropriately chosen so that the effective information rate is $40 \mathrm{~Gb} / \mathrm{s}$.

Fig. 6 shows the conditional PDFs $p\left(y_{j} \mid x[j-m, j+m]\right)$ obtained for dispersion map described above for $m=2$. As expected, by increasing the number of spans, the ghost pulse at the central bit position for the state $s={ }^{\prime} 11011^{\prime}$ grows, hence shifting the mean of the PDF to the right. After certain number of spans the mean of PDF exceeds the decision threshold resulting in an error. On the other hand, the mean of PDF for an isolated one (in state $s={ }^{\prime} 00100^{\prime}$ ) shifts to the left as the number of spans increases, suggesting that assumed
TABLE I

FIBER PARAMETERS

\begin{tabular}{|c|c|c|}
\hline Parameters & D $_{+}$FIBER & D_ FIBER $^{-}$ \\
\hline Dispersion $[\mathrm{ps} /(\mathrm{nm} \mathrm{km})]$ & 20 & -40 \\
\hline Dispersion Slope $\left[\mathrm{ps} /\left(\mathrm{nm}^{2} \mathrm{~km}\right)\right]$ & 0.06 & -0.12 \\
\hline Effective Cross-sectional Area $\left[\mu \mathrm{m}^{2}\right]$ & 110 & 50 \\
\hline Nonlinear refractive index $\left[\mathrm{m}^{2} / \mathrm{W}\right]$ & $2.6 \cdot 10^{-20}$ & $2.6 \cdot 10^{-20}$ \\
\hline Attenuation Coefficient $[\mathrm{dB} / \mathrm{km}]$ & 0.19 & 0.25 \\
\hline
\end{tabular}

memory $2 m+1=5$ is not sufficiently large to capture the effect of intersymbol interference completely. However, even the partial elimination of nonlinear ISI may lead to significant BER performance improvement, as shown later. To estimate the PDFs, the region of all possible samples is quantized in 64 bins, and the number of occurrences of samples in a given bin is counted and normalized with total number of samples in all bins.

The results of simulations for a single-channel optical transmission system operating at $40 \mathrm{~Gb} / \mathrm{s}$, with dispersion map described above, are shown in Fig. 7. The number of spans was changed from 20 to 60 in step of 2 , the uncoded BER at $40 \mathrm{~Gb} / \mathrm{s}$ (denoted as $B E R_{\text {unc }}$ ) and BER after iterative decoding at line rate $R_{l}=R_{b} / R\left(R_{b}=40\right.$ $\mathrm{Gb} / \mathrm{s}, R$ is the code rate) were calculated and given in Fig. 7 as $x-$ and $y$-axis respectively. In Fig. 7(a) we omitted the BCJR equalizer in order to be able to evaluate different classes of LDPC codes introduced in previous Section in the presence of bursts of errors due to intrachannel nonlinearities. The proposed LDPC codes outperform the TPC even in the presence of simultaneous random and bursts errors. The BER performance comparison of LDPC-coded TE against largegirth LDPC codes and turbo-product codes is given in Fig. 7(b), for different trellis memories. LDPC-coded TE with memory $2 m+1=7$ provides almost $12 \mathrm{~dB}$ improvement over memoryless BCJR equalizer (assumed memory is $m=0$ ) at BER of $10^{-8}$.

\section{Chromatic Dispersion Compensation}

The chromatic dispersion is time invariant so that it is quite straightforwardly to compensate for by using the advanced detection and coding techniques. Several of such techniques based on Viterbi equalizer have been recently proposed [7],[11].

The Viterbi equalizer is a maximum-likelihood sequence detection technique whose complexity grows exponentially with residual group-velocity dispersion. In order to reduce the complexity of Viterbi equalizer a narrowband electrical filtering has been proposed in [11], by exploiting the fact that duo-binary like modulation formats are more immune to residual chromatic dispersion than conventional NRZ modulation format. Nevertheless to achieve $600 \mathrm{~km}$ transmission without optical dispersion compensation Viterbi equalizer with 64 states was used. It has been shown in [7] that the Viterbi equalizer can be used to compensate for cumulative dispersion over $1000 \mathrm{~km}$, which represents an important theoretical result, 


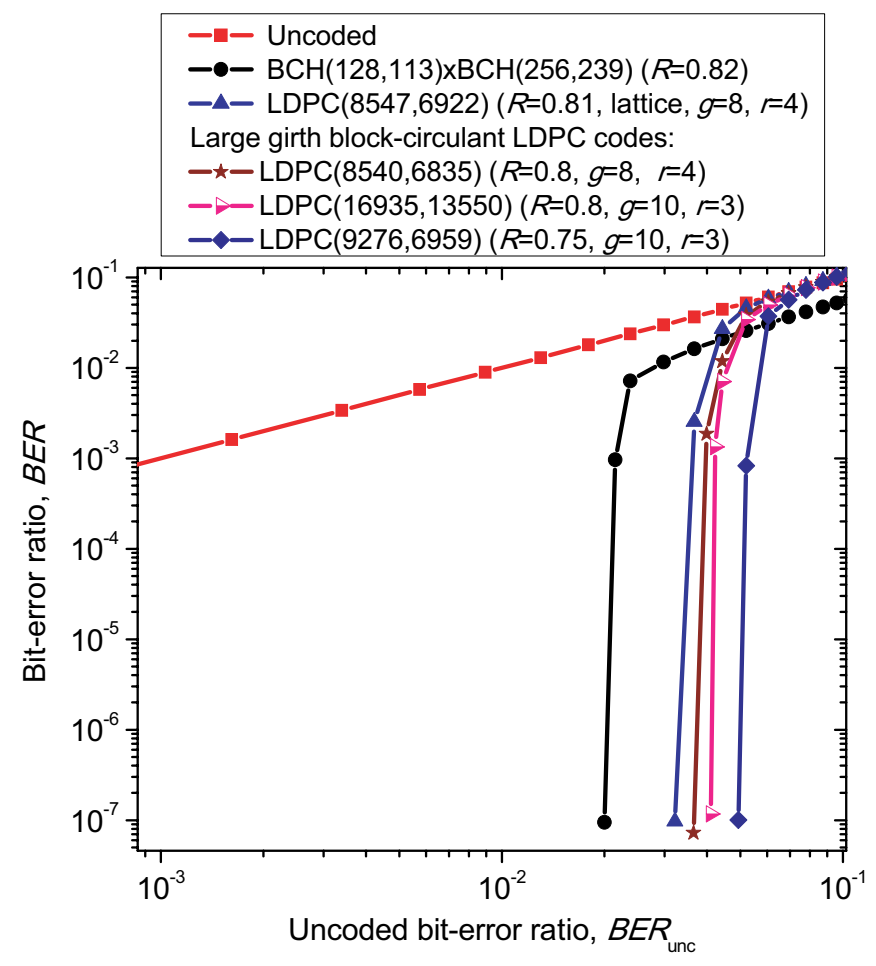

(a)

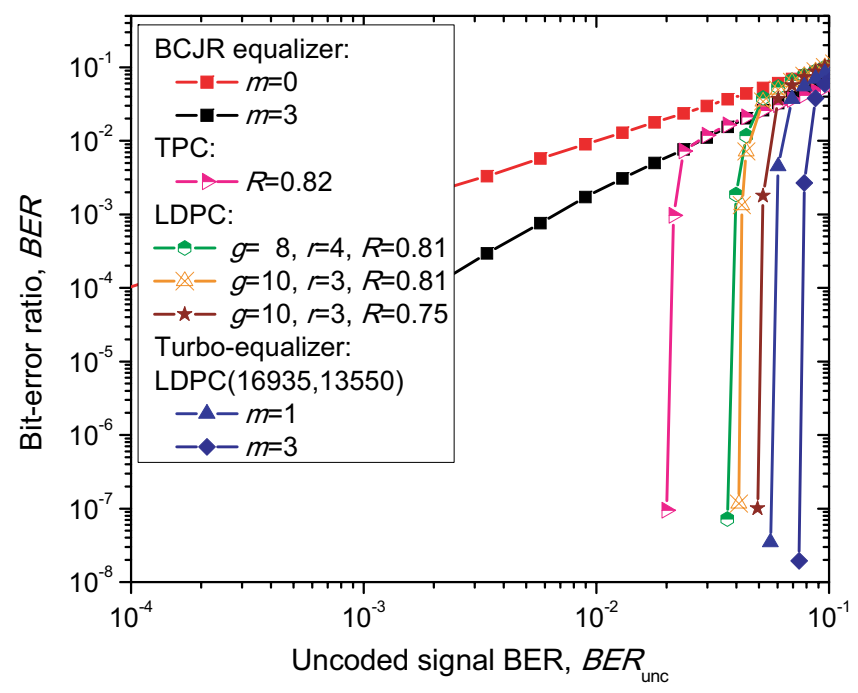

(b)

Fig. 7. BER performance of different coding schemes at $40 \mathrm{~Gb} / \mathrm{s}$ for dispersion map from Fig. 5: (a) BER performance of large girth blockcirculant LDPC codes, (b) LDPC-coded TE against LDPC codes and TPC.

but the complexity of Viterbi equalizer with 8192 states is too high to be of practical importance.

The simulations results for an NRZ single-channel optical transmission system given in Fig. 8, operating at 10 $\mathrm{Gb} / \mathrm{s}$ (effective information rate), are shown in Fig. 9. The dispersion and dispersion slope parameters are selected to be $16 \mathrm{ps} / \mathrm{nm}-\mathrm{km}$, and $0.08 \mathrm{ps} /\left(\mathrm{nm}^{2}-\mathrm{km}\right)$, respectively. NRZ pulses are modeled using raised-cosine pulse shape with rolloff factor of 0.5 , and the launched power is set to $0 \mathrm{dBm}$. The BCJR equalizer alone for trellis memory $2 m+1=7$ (128 states) enters the error floor, while TE provides more

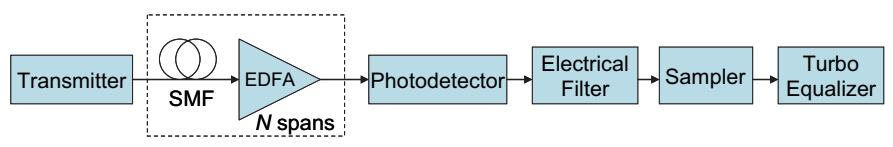

Fig. 8. System description for study of chromatic dispersion compensation based on LDPC-coded turbo equalization.

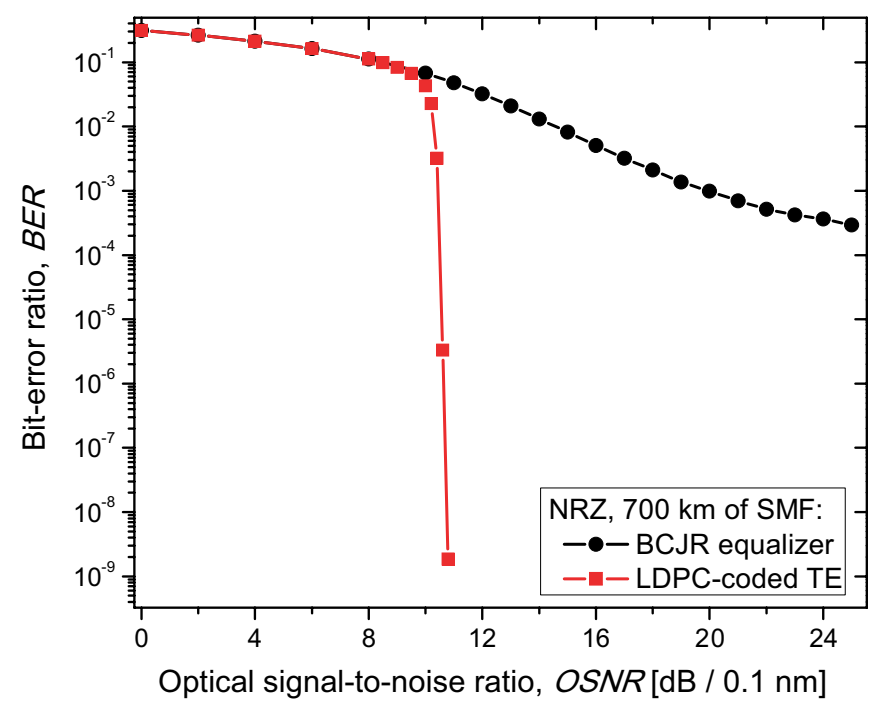

Fig. 9. BER performance of $\operatorname{LDPC}(16935,13550)$-coded chromatic dispersion turbo equalizer of trellis memory $2 m+1=7$ for SMF length of 700 $\mathrm{km}$.

than $700 \mathrm{~km}$ of error free transmission. Moreover, here we employed conventional NRZ instead of narrowband filtered NRZ used in [11].

\section{PMD COMPENSATION}

The nonlinear ISI TE introduced in Section II can also be used as a PMD compensator. As explained earlier, the TE (see Fig. 1(a)) is composed of two components: the BCJR equalizer, and LDPC decoder. The purpose of BCJR equalizer is to deal with ISI due to PMD, and to provide soft bit reliabilities (LLRs) for LDPC decoder. The results of simulations, for $10 \mathrm{~Gb} / \mathrm{s}$ (effective information rate) transmission and ASE noise dominated scenario, are shown in Fig. 10 for DGD $=100 \mathrm{ps}$ and girth-10 LDPC code of rate 0.81 . RZ-OOK of a duty cycle of 0.33 is observed. The bandwidth of optical filter (modeled as super-Gaussian of order 8 ) is set to $3 R_{l}$, and the bandwidth of electrical filter (modeled as Gaussian) to $0.7 R_{l}$, with $R_{l}$ being the line rate. For DGD of $100 \mathrm{ps,}$ the $R=0.81$ LDPC-coded turbo equalizer (for trellis memory $2 m+1=7$ ) has penalty of only $2 \mathrm{~dB}$ with respect to the backto-back configuration. The turbo equalizer employing the code of girth-10 provides more than $1 \mathrm{~dB}$ improvement with respect to girth-8 LDPC code we employed in [10]. (Notice however, that scheme in [10] operates at $40 \mathrm{~Gb} / \mathrm{s}$.)

We further demonstrate the capability of LDPC-coded turbo equalizer in simultaneous chromatic dispersion and PMD compensation. The results of simulation after $700 \mathrm{~km}$ of SMF and for DGD of $50 \mathrm{ps}$, assuming NRZ transmission at 10 $\mathrm{Gb} / \mathrm{s}$, are given in Fig. 11. The BCJR equalizer enters BER floor that is so high that even concatenated RS code is not 


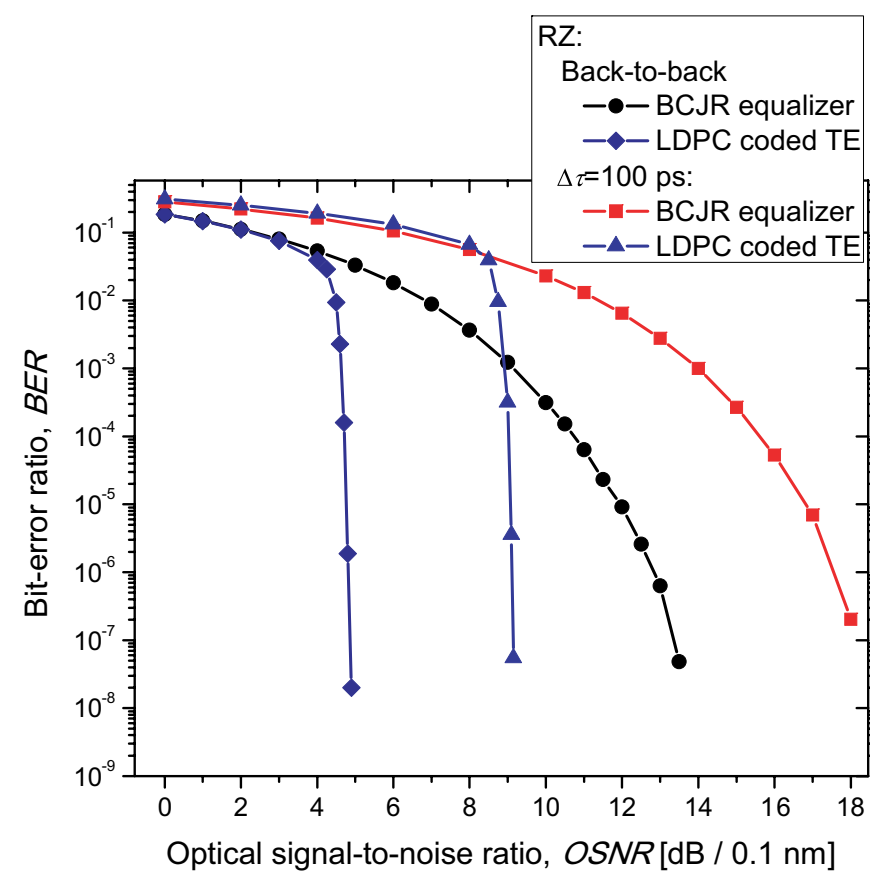

Fig. 10. BER performance of $\operatorname{LDPC}(16935,13550)$-coded PMD TE with trellis memory $2 m+1=7$.

able to handle, while LDPC-coded turbo equalizer is able to operate properly.

In the rest of this Section we turn our attention to the experimental verification. The experimental setup for PMD compensation study by LDPC-coded turbo equalization is shown in Fig. 12. The LDPC-encoded sequence is uploaded into Anritsu pattern generator via GPIB card controlled by a PC. A zero-chirp Mach-Zehnder modulator is used to generate the NRZ data stream. The launch power is maintained at $0 \mathrm{dBm}$ at the input of PMD emulator (with equal power distribution between states of polarization). The output of PMD emulator is combined with an ASE source immediately prior to the preamplifier. The ASE noise power is controlled by variable optical attenuator (VOA) in order to provide an independent optical SNR (OSNR) adjustment at the receiver. A standard pre-amplified PIN receiver is used for direct detection and is preceded by another VOA to maintain a constant received power of $-6 \mathrm{dBm}$. The sampling oscilloscope (Agilent), triggered by the data pattern, is used to acquire the received sequences, downloaded via GPIB card back to the PC which serves as an LDPC-coded turbo equalizer. The experimental results for 10 Giga symbols/s NRZ transmission are shown in Fig. 13, for different DGD values. The TE is based quasi-cyclic $\operatorname{LDPC}(11936,10819)$ code of code rate 0.906 and girth-10, with 5 outer and 25 sum-product algorithm iterations. The OSNR penalty for DGD of $125 \mathrm{ps}$ is about $3 \mathrm{~dB}$ at $B E R=10^{-6}$, while the coding gain improvement over BCJR equalizer (with memory $2 m+1=5$ ) for DGD $=125 \mathrm{ps}$ is $6.25 \mathrm{~dB}$ at $B E R=10^{-6}$. Larger coding gains are expected at lower BERs.

In previous three Sections we have demonstrated that LDPC-coded turbo equalizer is an excellent nonlinear ISI equalizer, capable to simultaneously mitigate intra-channel nonlinearities, residual chromatic dispersion and PMD. This

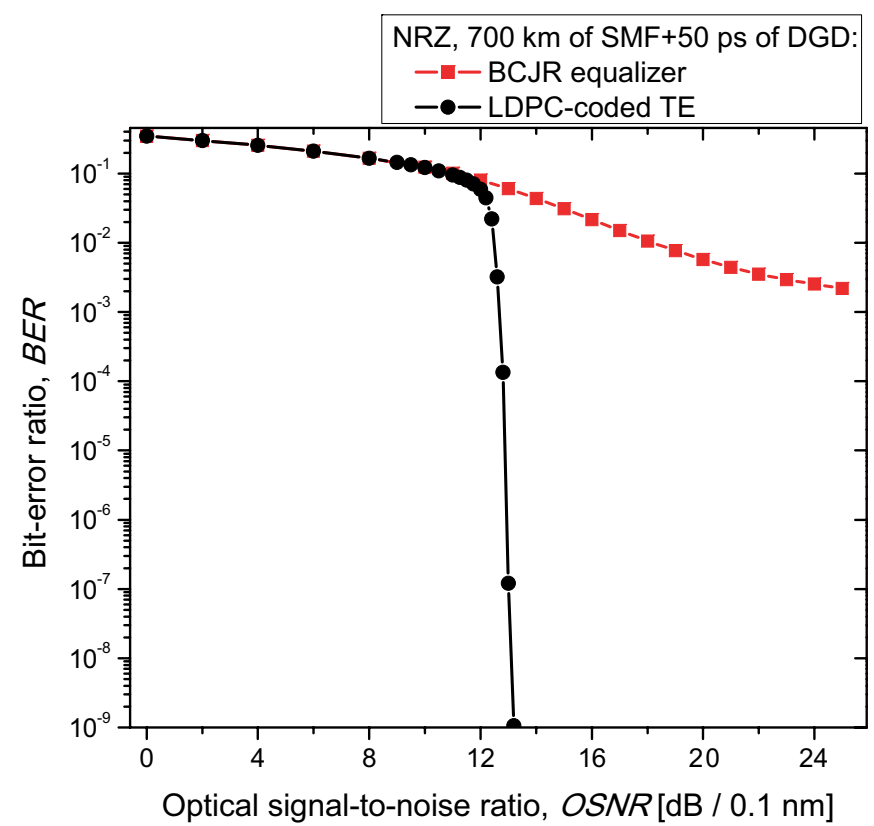

Fig. 11. BER performance of $\operatorname{LDPC}(16935,13550)$-coded TE of trellis memory $2 m+1=7$ for simultaneous chromatic dispersion and PMD compensation.

lead us to a non-trivial question, what is the channel capacity and how closely can it be approached by using LDPC-coded turbo equalization. This problem is addressed in next Section.

\section{Achievable Information Rate Study}

In this Section we address the problem of calculating the channel capacity for an i.i.d. information source. The i.i.d. channel capacity is also known as achievable information rate (see [26]-[29] and references therein), and represents the lower bound on channel capacity. To calculate the i.i.d. channel capacity, similarly as in Section II, we model the optical channel (the whole transmission system) as the nonlinear ISI channel, in which $m$ previous and next $m$ bits influence the observed bit. The optical communication channel is characterized by the conditional PDF of the output vector of samples $y=\left(y_{1}, \ldots, y_{n}, \ldots\right), y_{i} \in Y$ ( $Y$ is the set possible outputs), given the source (channel input) sequence $x=\left(x_{1}, \ldots, x_{n}, \ldots\right), x_{i} \in X=\{0,1\}$. The information rate can be calculated from:

$$
I(Y ; X)=H(Y)-H(Y \mid X),
$$

where $H(U)=E\left(\log _{2} P(U)\right)$ is the entropy of a random variable $U$ and $E()$ is the mathematical expectation operator. The Shannon-McMillan-Brieman theorem states that [30]:

$$
E\left(\log _{2}(P(Y))\right)=\lim _{n->\infty}\left[(1 / n) \log _{2}(P(y[1, n]))\right],
$$

where $y[1, n]=\left(y_{1}, \ldots, y_{n}\right)$. Thus the information rate can be determined by calculating $\log _{2}(P(y[1, n]))$, for sufficiently long sequence. By substituting (10) into (9) we get (11). Given the channel model, the conditional PDF described in Section II $P\left(y_{i} \mid y[1, i-1], x[1, n]\right)=P\left(y_{i} \mid x[i-m, i+m]\right)=P\left(y_{i} \mid s\right)$. Therefore, the first term in (11) can be straightforwardly calculated from transition PDFs $P\left(y_{i} \mid s\right)$. To calculate $P\left(y_{i} \mid y[1, i-\right.$ $1]$ ) we use the forward recursion of BCJR algorithm described 


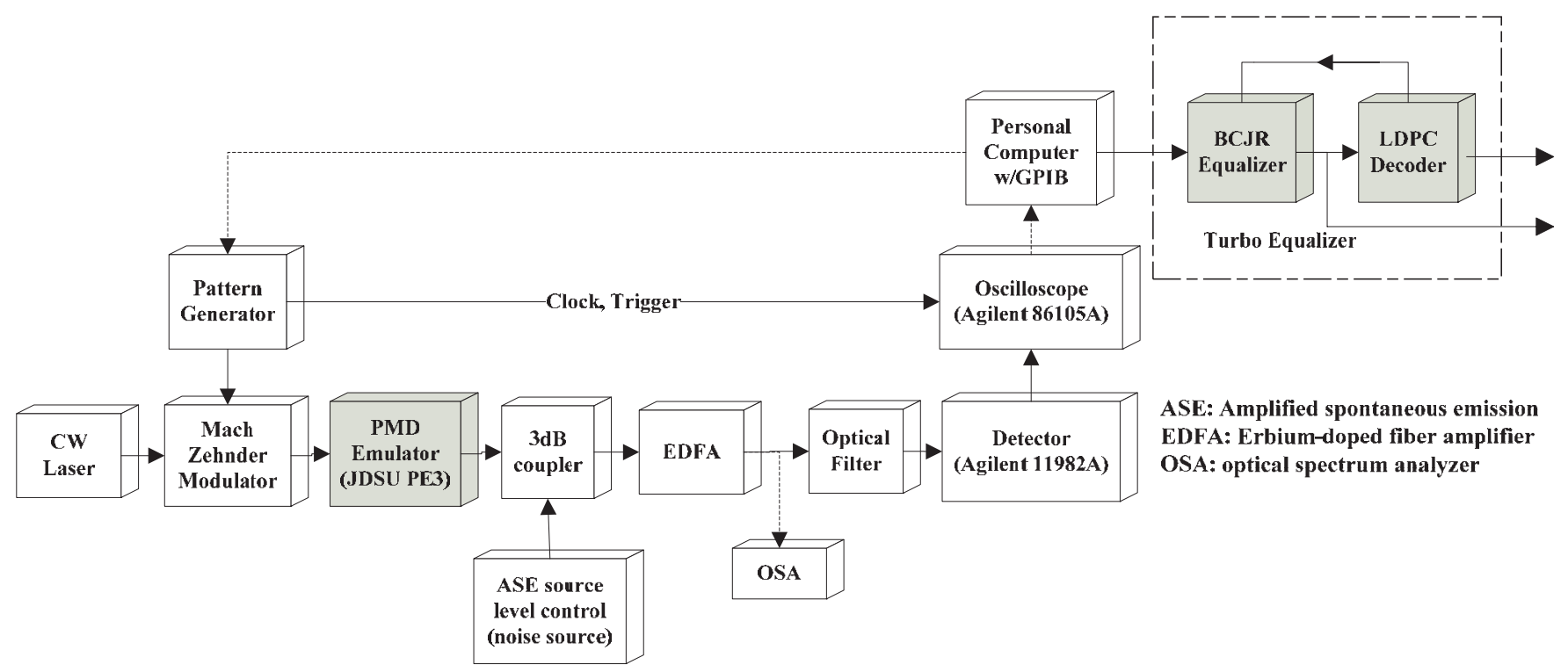

Fig. 12. Experimental setup for PMD compensation study by LDPC-coded turbo equalization.

$$
I(Y ; X)=\lim _{n->\infty}\left[(1 / n) \sum_{i=1}^{n} \log _{2}\left(P\left(y_{i} \mid y[1, i-1], x[1, n]\right)\right)-(1 / n) \sum_{i=1}^{n} \log _{2}\left(P\left(y_{i} \mid y[1, i-1]\right)\right)\right] .
$$

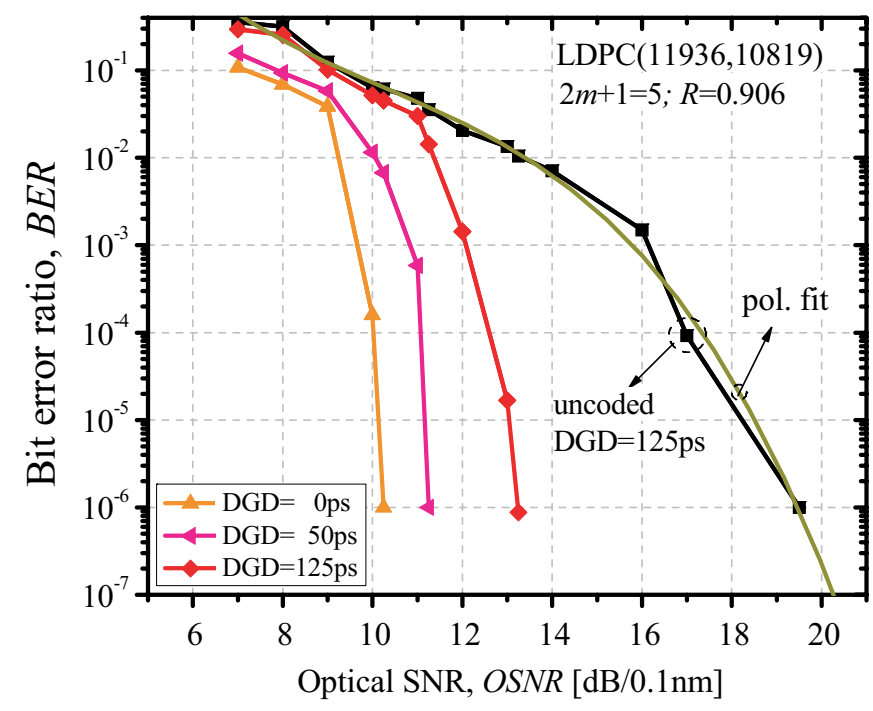

Fig. 13. Experimentally obtained BERs of LDPC(11936,10819)-coded PMD compensator.

in Section II. Notice that in [26],[27] we used the forward recursion of probabilistic-domain BCJR algorithm to calculate the i.i.d. channel capacity. In this paper, we use the logdomain version instead (similarly as in [28],[29]), because it is numerically more stable and allows to study the quantization effects. Moreover, the references [26],[27] concentrate on fiber nonlinearities, the reference [28] on chromatic dispersion and the reference [29] on PMD. Here we consider the joint influence of different channel impairments on i.i.d. channel capacity.

In Fig. 14 we show the i.i.d. channel capacity against optical SNR for different memory assumptions in BCJR equalizer,

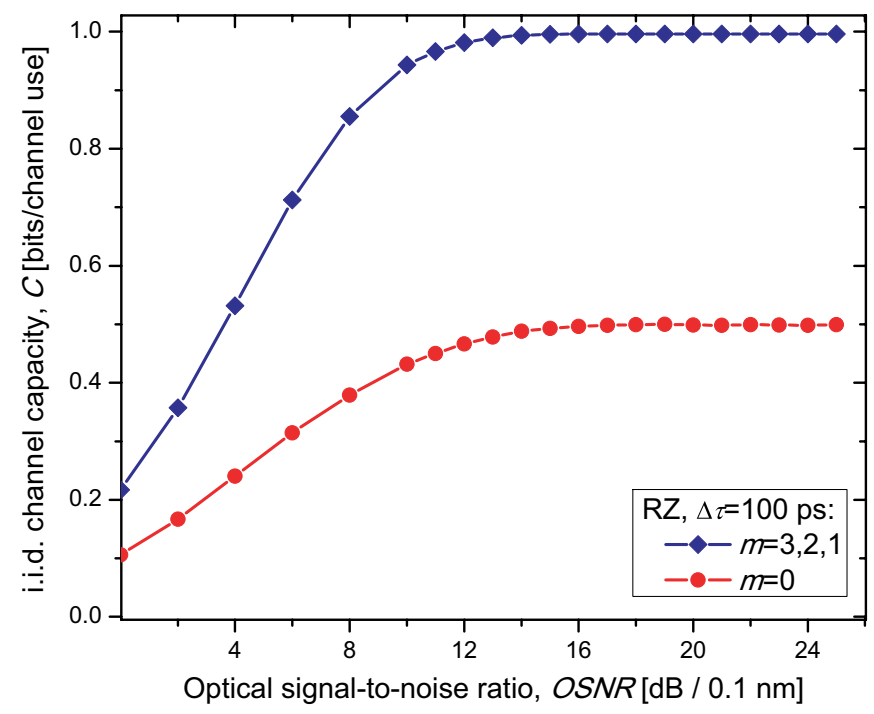

Fig. 14. i.i.d. channel capacity for DGD of $100 \mathrm{ps,}$ assuming RZ transmission at $10-\mathrm{Gb} / \mathrm{s}$.

in the presence of PMD, for DGD of 100 ps, observing the RZ transmission at $10 \mathrm{~Gb} / \mathrm{s}$. As expected, the BCJR equalizer of memory $m=1$ is sufficient to compensate for this level of DGD, while the memoryless assumption $(m=0)$ leads to significant i.i.d. channel capacity loss. In Fig. 15 we show the i.i.d. channel capacity against optical SNR for different memory assumptions in BCJR equalizer, in the presence of residual chromatic dispersion of 11200 $\mathrm{ps} / \mathrm{nm}$ (which corresponds to $700 \mathrm{~km}$ of SMF with dispersion and dispersion slope being $16 \mathrm{ps} / \mathrm{nm}-\mathrm{km}$, and $0.08 \mathrm{ps} / \mathrm{nm}^{2}$ $\mathrm{km}$, respectively). The NRZ transmission system operating at $10 \mathrm{~Gb} / \mathrm{s}$ is observed. The BCJR equalizer memory $m=3$ is 


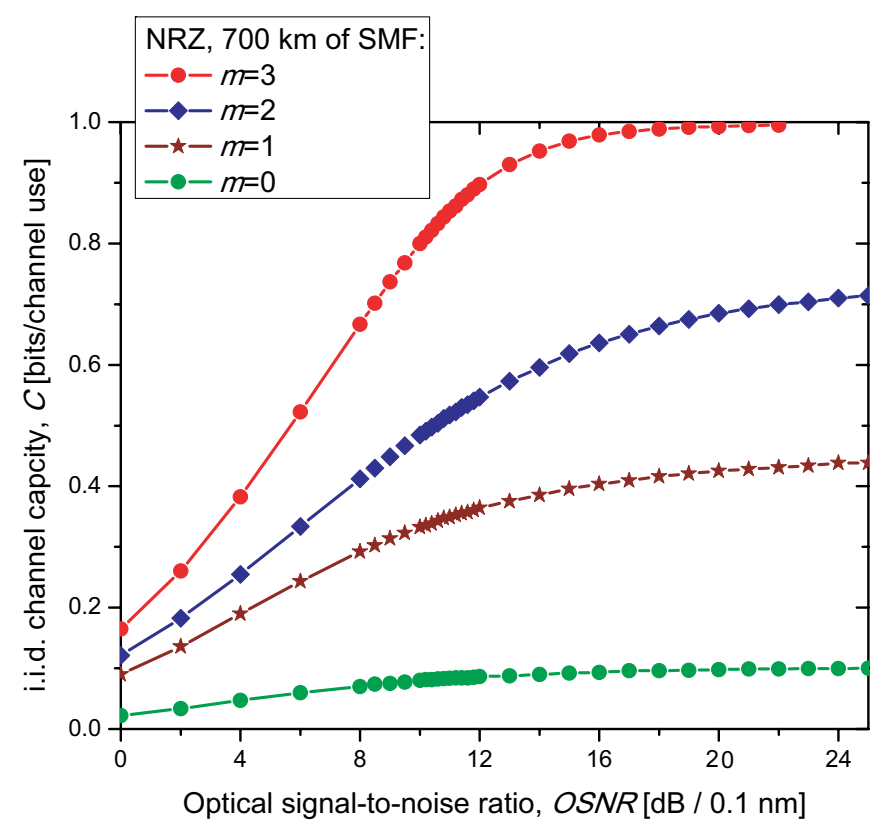

Fig. 15. i.i.d. channel capacity after $700 \mathrm{~km}$ of SMF, assuming NRZ transmission at $10-\mathrm{Gb} / \mathrm{s}$.

sufficient to compensate for this level of residual chromatic dispersion, while for the lower memories this effect cannot be eliminated at all by any equalization scheme alone, and FEC is unavoidable. To achieve the i.i.d. channel capacity of 1, for lower optical SNR values, we have to increase the BCJR equalizer memory. In Fig. 16 we show the i.i.d. channel capacity against optical SNR for different memories in BCJR equalizer, in the presence of both residual chromatic dispersion (11200 ps/nm) and PMD with DGD of $50 \mathrm{ps.} \mathrm{The}$ NRZ transmission system operating at $10 \mathrm{~Gb} / \mathrm{s}$ is observed as well. For the memory $m=3$, the unity information rate is achieved for higher OSNR values (about $4 \mathrm{~dB}$ higher). The LDPC code of rate 0.8 used in Fig. 11 is only $1 \mathrm{~dB}$ away from the channel capacity. To come closer to the channel capacity curve we have to increase the number of iterations, both inner and outer ones, and to employ longer LDPC codes. In Fig. 17 we show the i.i.d. channel capacity in the presence of intrachannel nonlinearities against the number of spans, for dispersion map shown in Fig. 5. The RZ transmission system operating at $40 \mathrm{~Gb} / \mathrm{s}$ is observed. For this particular dispersion map, if LDPC codes of rate 0.8 are used, and assuming that the BCJR equalizer operates with memory $m=3,7200 \mathrm{~km}$ can be achieved. To extend the transmission distance further, we have to increase the memory in trellis description of channel, employ an optimum dispersion map, and optimize the system parameters. In Fig. 18 we show how the i.i.d. channel capacity degrades with quantization of LLRs, for the same system used in Fig. 17. The use of three bits to represent the LLRs result in moderate channel capacity loss, while the use of only 2 bits results in significant channel capacity loss.

\section{SUMMARY}

In this paper we studied the LDPC-coded turbo equalization scheme as a universal nonlinear ISI equalizer that can be used to simultaneously suppress fiber nonlinearities, and to compen-

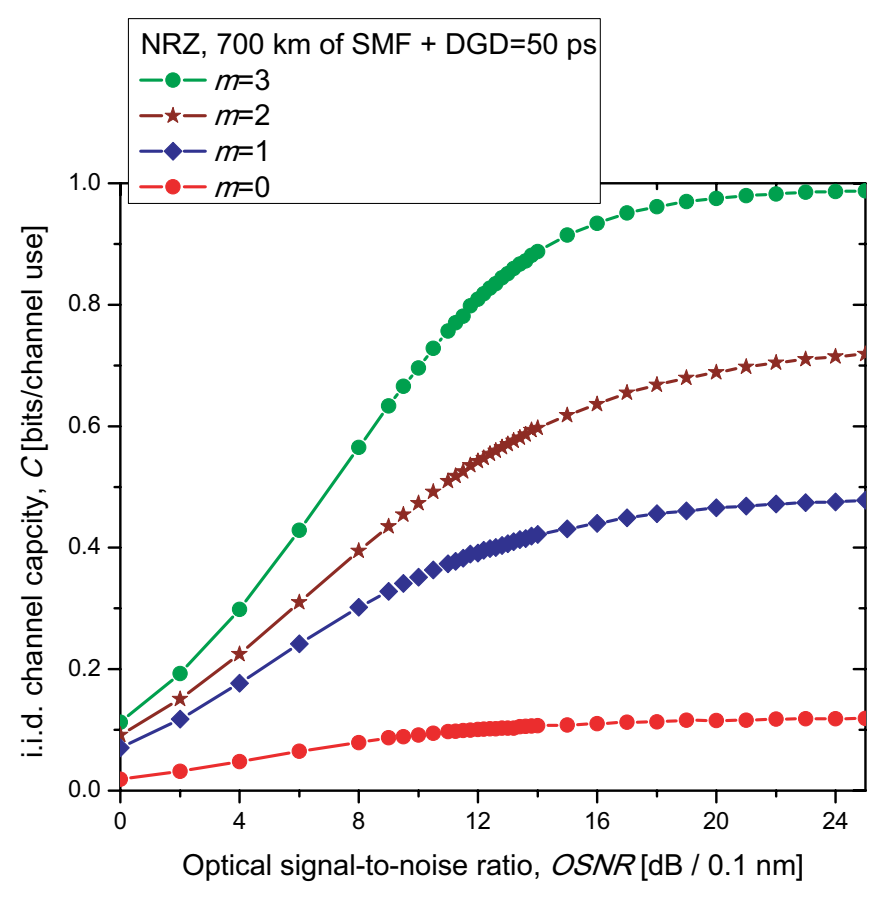

Fig. 16. i.i.d. channel capacity after $700 \mathrm{~km}$ of SMF and for DGD of 50 ps, assuming NRZ transmission at $10-\mathrm{Gb} / \mathrm{s}$.

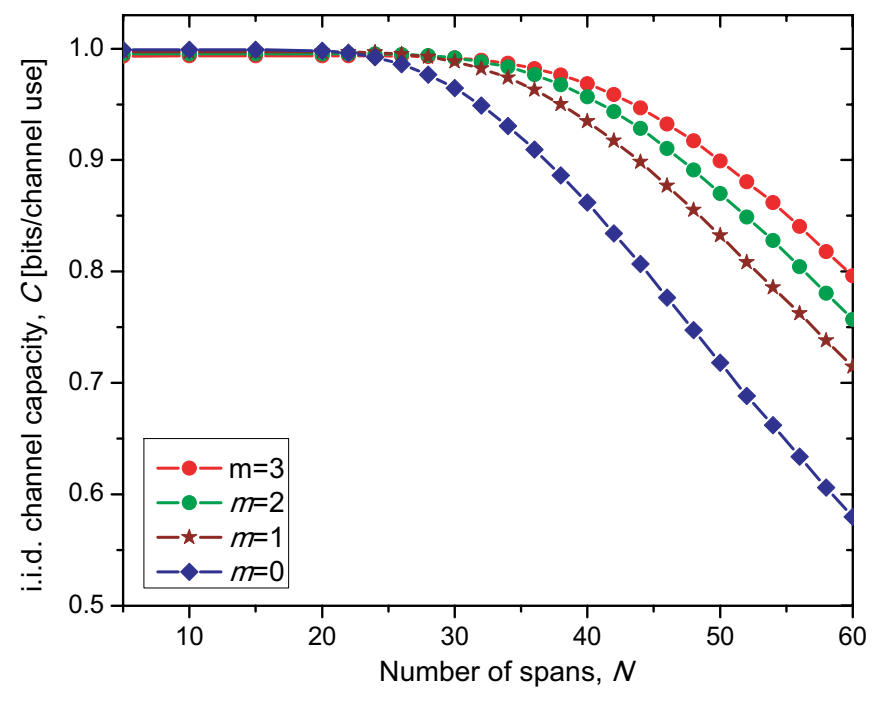

Fig. 17. The influence of memory effects on i.i.d. channel capacity versus the number of spans for dispersion map shown in Fig. 5, assuming RZ transmission at $40-\mathrm{Gb} / \mathrm{s}$.

sate accumulated chromatic dispersion and PMD. The LDPC codes required for turbo equalizer were designed as large girth $(g \geq 8)$ block-circulant (array) LDPC codes, and the MAP detector was implemented using BCJR algorithm based equalizer. The LDPC codes from this class have low complexity of decoder, and are suitable for FPGA and VLSI implementations at high-speed. The first step toward the FPGA implementation of LDPC-coded turbo-equalizer, the FPGA implementation of quasi-cyclic LDPC decoder has been described in our recent paper [31]. For an NRZ system operating at $10 \mathrm{~Gb} / \mathrm{s}$ with residual dispersion of $11200 \mathrm{ps} / \mathrm{nm}$ and for DGD of 50 ps, the LDPC-coded TE operating on trellis with memory $m=3$ is only $1 \mathrm{~dB}$ away from the i.i.d channel capacity. 


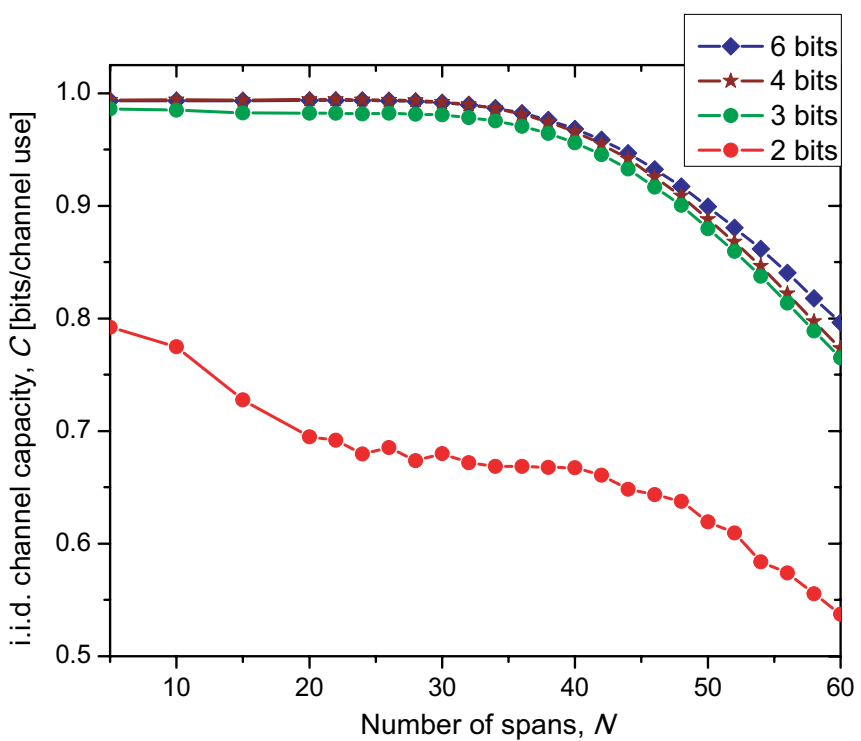

Fig. 18. i.i.d. channel capacity loss due to quantization of LLRs versus the number of spans for dispersion map shown in Fig. 5, assuming RZ transmission at $40-\mathrm{Gb} / \mathrm{s}$.

The LDPC-coded TE provides almost $12 \mathrm{~dB}$ improvement over BCJR equalizer for an RZ system operating at $40 \mathrm{~Gb} / \mathrm{s}$ in the presence of intrachannel nonlinearities. We perform simulations to demonstrate that this turbo equalizer is able to simultaneously deal with different channel impairments. We conduct experiments to verify the efficiency of this equalizer in PMD compensation. We determined the i.i.d. channel capacity, and studied how closely the channel capacity can be approached using turbo equalizer of reasonable complexity.

\section{REFERENCES}

[1] R.-J. Essiambre, G. Raybon, and B. Mikkelsen, "Pseudo-linear transmission of high-speed TDM signals 40 and $160 \mathrm{~Gb} / \mathrm{s}, "$ in Optical Fiber Telecommunications IVB ed. by I. P. Kaminow and T. Li, p. 233-304, San Diego, CA: Academic Press, 2002.

[2] X. Liu et al., "Suppression of interchannel four-wave-mixing-induced ghost pulses in high-speed transmissions by phase inversion between adjacent marker blocks," Opt. Lett., vol. 27, pp. 1177-1179, July 2002.

[3] I. B. Djordjevic, "Suppression of intrachannel nonlinearities in highspeed WDM systems," in Advanced Technologies for High-Speed Optical Communications ed. by L. Xu, p. 247-277, Trivandrum-Kerala, India: Research Signpost, 2007.

[4] H. Blow, F. Buchali, and B. Franz, "Enhancement of tolerances in transmission systems by analog and digital signal processing," AsiaPacific Optical Communications Conference (APOC 2005), Nov. 2005, Shanghai, China, in Proc. SPIE, vol. 6021, paper 6021-163.

[5] M. Jger, T. Rankl, J. Spiedel, H. Bulw, and F. Buchali, "Performance of turbo equalizers for optical PMD channels," J. Lightwave Technol., vol. 24, pp. 1226-1236, Mar. 2006.

[6] I. B. Djordjevic et al., "Low-density parity-check codes for $40 \mathrm{~Gb} / \mathrm{s}$ optical transmission systems," IEEE J. Select. Top. Quantum Electron., vol. 12, pp. 555-562, July/Aug. 2006.

[7] P. Poggiolini, G. Bosco, S. Savory, Y. Benlachtar, R. I. Killey, and J. Prat, "1,040 km uncompensated IMDD transmission over G.652 fiber at $10 \mathrm{Gbit} / \mathrm{s}$ using a reduced-state SQRT-metric MLSE receiver," in Proc. ECOC 2006, post-deadline paper Th4.4.6, Sept. 2006, Cannes, France.

[8] I. B. Djordjevic and B. Vasic, "Nonlinear BCJR equalizer for suppression of intrachannel nonlinearities in $40 \mathrm{~Gb} / \mathrm{s}$ optical communications systems," Opt. Express, vol. 14, pp. 4625-4635, May 2006.

[9] H. G. Batshon, I. B. Djordjevic, and L. Minkov, "Chromatic dispersion compensation using LDPC-coded turbo equalization," in Proc. IEEE LEOS Summer Topicals 2007: Advanced Digital Signal Processing in Next Generation Fiber Optic Transmission, pp. 37-38, July 2007, Portland, OR, USA.
[10] I. B. Djordjevic, H. G. Batshon, M. Cvijetic, L. Xu, and T. Wang, "PMD compensation by LDPC-coded turbo equalization," IEEE Photon. Technol. Lett., vol. 19, no. 15, pp. 1163-1165, Aug. 1, 2007.

[11] N. Alic, G. C. Papen, R. E. Saperstein, R. Jiang, C. Marki, Y. Fainman, and S. Radic, "Experimental demonstration of $10 \mathrm{~Gb} / \mathrm{s}$ NRZ extended dispersion-limited reach over 600km-SMF link without optical dispersion compensation," in Proc. Optical Fiber Communication Conference, 2006 and the 2006 National Fiber Optic Engineers Conference, Mar. 2006, paper no. OWB7.

[12] D. J. C. MacKay, "Good error correcting codes based on very sparse matrices," IEEE Trans. Inform. Theory, vol. 45, pp. 399-431, 1999.

[13] M. P. C. Fossorier, "Quasi-cyclic low-density parity-check codes from circulant permutation matricies," IEEE Trans. Inform. Theory, vol. 50, pp. 1788-1794, Aug. 2004.

[14] L. R. Bahl, J. Cocke, F. Jelinek, and J. Raviv, "Optimal decoding of linear codes for minimizing symbol error rate," IEEE Trans. Inform. Theory, vol. IT-20, pp. 284-287, Mar. 1974.

[15] H. Xiao-Yu, E. Eleftheriou, D.-M. Arnold, and A. Dholakia, "Efficient implementations of the sum-product algorithm for decoding of LDPC codes," in Proc. IEEE Globecom 2001, vol. 2, Nov. 2001, pp. 10361036E.

[16] C. Douillard, M. Jzquel, C. Berrou, A. Picart, P. Didier, and A. Glavieux, "Iterative correction of intersymbol interference: turbo equalization," Eur. Trans. Telecommun., vol. 6, pp. 507-511, 1995.

[17] S. ten Brink, "Convergence behavior of iteratively decoded parallel concatenated codes," IEEE Trans. Commun., vol. 40, pp. 1727-1737, Oct. 2001

[18] M. Ivkovic, I. Djordjevic, P. Rajkovic, and B. Vasic, "Pulse energy probability density functions for long-haul optical fiber transmission systems by using instantons and Edgeworth expansion," IEEE Photon. Technol. Lett., vol. 19, no 20, pp. 1604-1606, Oct. 15, 2007.

[19] W. E. Ryan, "Concatenated codes and iterative decoding," in Wiley Encyclopedia of Telecommunications (J. G. Proakis, ed.), New York: Wiley and Sons, 2003.

[20] B. M. Kurkoski, P. H. Siegel, and J. K. Wolf, "Joint message-passing decoding of LDPC codes and partial-response channels," IEEE Trans. Inform. Theory, vol. 48, pp. 1410-1422, June 2002.

[21] I. B. Djordjevic, M. Cvijetic, L. Xu, and T. Wang, "Using LDPCcoded modulation and coherent detection for ultra high-speed optical transmission," IEEE/OSA J. Lightwave Technol., vol. 25, pp. 3619-3625, Nov. 2007.

[22] R. M. Tanner, "A recursive approach to low complexity codes," IEEE Trans. Inform. Theory, vol. IT-27, no. 5, pp. 533-547, Sept. 1981.

[23] O. Milenkovic, I. B. Djordjevic, and B. Vasic, "Block-circulant lowdensity parity-check codes for optical communication systems," IEEE $J$. Select. Top. Quantum Electron., vol. 10, no. 2, pp. 294-299, Mar./Apr. 2004.

[24] J. L. Fan, "Array codes as low-density parity-check codes," in Proc. 2nd Int. Symp. Turbo Codes and Related Topics, Brest, France, pp. 543-546, Sept. 2000.

[25] T. Mizuochi et al., "Forward error correction based on block turbo code with 3-bit soft decision for 10-Gb/s optical communication systems," IEEE J. Select. Topics Quantum Electron., vol. 10, no. 2, pp. 376-386, Mar./Apr. 2004.

[26] I. B. Djordjevic, B. Vasic, M. Ivkovic, and I. Gabitov, "Achievable information rates for high-speed long-haul optical transmission," IEEE/OSA J. Lightwave Technol., vol. 23, pp. 3755-3763, Nov. 2005.

[27] M. Ivkovic, I. B. Djordjevic, and B. Vasic, "Calculation of achievable information rates of long-haul optical transmission systems using instanton approach," IEEE/OSA J. Lightwave Technol., vol. 25, pp. 1163-1168, May 2007.

[28] I. B. Djordjevic, N. Alic, G. Papen, and S. Radic, "Determination of achievable information rates (AIRs) of IM/DD systems and AIR loss due to chromatic dispersion and quantization," IEEE Photon. Technol. Lett., vol. 19, no. 1, pp. 12-14, Jan. 1, 2007.

[29] L. L. Minkov, I. B. Djordjevic, H. G. Batshon, L. Xu, T. Wang, M. Cvijetic, and F. Kueppers, "Demonstration of PMD compensation by LDPCcoded turbo equalization and channel capacity loss characterization due to PMD and quantization," IEEE Photon. Technol. Lett., vol. 19, no. 22, pp. 1852-1854, Nov. 15, 2007.

[30] T. M. Cover, Elements of Information Theory. New York: Wiley, 1991.

[31] M. Arabaci, I. B. Djordjevic, and R. Saunders, "FPGA implementation of LDPC decoders for large girth LDPC codes," in preparation. 


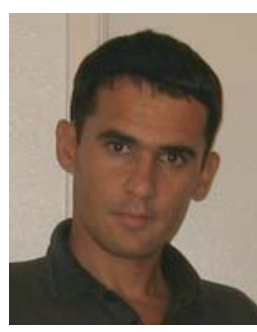

Ivan B. Djordjevic is an Assistant Professor of Electrical and Computer Engineering at the University of Arizona, Tucson. Prior to this appointment in August 2006, he was with University of Arizona, Tucson, USA; University of the West of England, Bristol, UK; University of Bristol, Bristol, UK; Tyco Telecommunications, Eatontown, USA; and National Technical University of Athens, Athens, Greece. His current research interests include optical networks, error control coding, constrained coding, coded modulation, turbo equalization, OFDM applications, and quantum communications. Dr. Djordjevic is author of more than 100 international publications. He presently directs the Optical Communications Systems Laboratory (OCSL) within the ECE Department at the University of Arizona and serves as an Associate Editor for RESEARCH LETTERS IN OPTICS.

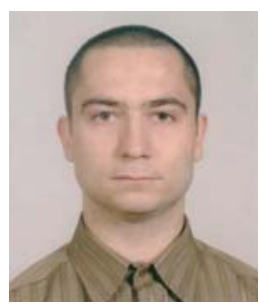

Lyubomir L. Minkov received a Master's degree in electrical engineering from the Technical University of Sofia, Bulgaria. Currently he is is a $\mathrm{Ph} . D$. student at the University of Arizona, Electrical and Computer Engineering Department. His current research interests include application of error correction coding in optical communication systems and modulation schemes in optical communications.

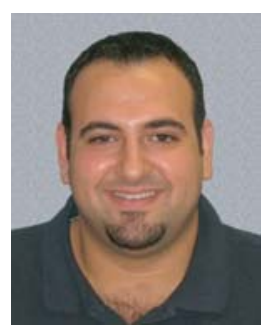

Hussam G. Batshon is currently a Ph.D. student at the Department of Electrical and Computer Engineering, University of Arizona, USA, where he has also received his M.S. degree in 2007. He was a Fulbright Scholarship grantee for the period 2005 - 2007. His current research interests include advanced coding and modulation schemes in addition to signal equalization and compensation for optical communication systems. Further information about his research work can be found at http://ece.arizona.edu/ hbatshon 